\section{THE IMPLEMENTATION OF CLINICAL GUIDELINES CONCERNING RETURN TO WORK AFTER MYOCARDIAL INFARCTION}

\begin{abstract}
1,2Shlomo Moshe, 2,3 Oren Zack, ${ }^{2,4}$ Miri Tzuberi, ${ }^{2,5}$ Gabriel Hodik. 'Maccabi Healthcare Services, Occupational Department, Holon, Israel; ${ }^{2}$ Sackler Faculty of Medicine, School of Public Health, Division of Environmental and Occupational Medicine, Tel-Aviv University. TelAviv, Israel; ${ }^{3}$ srael Defence Force Medical Force, Ramat Gan, Israel; ${ }^{4}$ Maccabi Healthcare Services, Department of Physiotherapy, Central District, Tel-Aviv, Israel; ${ }^{5}$ Maccabi Healthcare Services, Central Headquarter, Tel-Aviv, Israel
\end{abstract}

\subsection{6/oemed-2018-ICOHabstracts. 182}

Objectives The clinical guidelines concerning return to work after myocardial infarction (MI) were published in Israel on 2003. These guidelines were further published in the local medical association journal, Harefua, 2007. Our purpose was to examine the implementation of these guidelines by Occupational Physicians (OP).

Methods In this retrospective study, we analysed medical records of patients diagnosed with recent MI. We collected medical records data on 3 populations:

- before the publication (2000-2003);

- after the first publication (2004-2007) and

- following the review article publication (2008-2011).

Results We found improvement in medical history records on the following parameters: documenting years of education (18\%, 45\%, 56\%, p <0.001); occupation (87\%, 98\%, 100\%, $\mathrm{p}<0.001)$; length of hospitalisation $(80 \%, 95 \%$, and $94 \%$, $\mathrm{p}<0.001)$; complications during hospitalisation $(24 \%, 69 \%$, $73 \%, \quad \mathrm{p}<0.001)$; comorbidities $(65 \%, 94 \%$ and $97 \%$, $\mathrm{p}<0.001)$; NYHA classification $(70 \%, 81 \%$, and $92 \%$, $\mathrm{p}<0.001)$; and the scope of stress test $(51 \%, 79 \%$, and $89 \%$, $\mathrm{p}<0.001$ ); all of the above parameters correspond to populations 1,2 and 3 respectively. When it came to determining working capacity and recommendation of sick leave, we found a weak correlation with different parameters, with no significant difference between populations.

Conclusions This is the first study, which evaluates the implementation of clinical guidelines using clinical variables taken from medical records, and over a period of 12 years. Most of the required parameters based on the guidelines were collected indicating a good implementation. However, we found a weaker implementation concerning sick leave guidelines. We assume that more resources and activity are needed in order to achieve better compliance.

\section{A CROSS SECTIONAL STUDY OF PREVALENCE OF DEPRESSION, ANXIETY AND STRESS AMONGST PROFESSIONAL CAB DRIVERS IN NEW DELHI}

${ }^{1}$ A Rathi*, ${ }^{1}$ V Kumar, ${ }^{1} \mathrm{P}$ Lal, ${ }^{2} \mathrm{~A}$ Singh. ${ }^{1}$ Dr Baba Saheb Ambedkar Medical College and Hospital, New Delhi, India; ${ }^{2}$ Ministry of Health and Family Welfare, New Delhi, India

\subsection{6/oemed-2018-ICOHabstracts. 183}

Introduction In recent years, professional cab driving has seen a revolution with the advent of application based cab services, which are extremely time-bound. This poses a great burden on the service providers - the cab drivers. With over 70000 vehicles running as cabs, a good number of accidents, road rage cases and traffic violations involve the same. The mental health of drivers plays a key role in the increasing menace on road.
Methods A cross sectional study conducted on 111 cab drivers between February to May 2017. Their distress level was measured with the help of DASS-21 (Depression Anxiety Stress Scale) questionnaire. Data was analysed using SPSS.

Result In the current study, 55.9\% drivers worked for more than 12 hours a day, $31.5 \%$ worked on all 7 days of a week and only $39.6 \%$ get the required 8 hours of sleep on most days. Around $12 \%$ of the drivers have been involved in a road rage incident, $50 \%$ have been booked for violating traffic rules and $25 \%$ have been involved in minor accidents. Notably, one-fourth of the drivers are irritated while driving. It was noted that $53.2 \%$ had some degree of depression, with $19.8 \%$ having severe and $5.4 \%$ having extremely severe depression. Additionally, $36.9 \%$ and $32.4 \%$ drivers also had varied degrees of anxiety and stress, respectively. Pearson correlation test shows that DAS score increases as duration of sleep decreases. Significant association was also seen between the irritation of drivers and working hours of more than 12 hours $\left(\chi^{2}=8.76, \mathrm{p}\right.$ value $\left.=0.003\right)$.

Discussion Majority of cab drivers are suffering from physical and mental morbidities. There is a need to address to their healthcare status by the companies that have hired them. Rules should be made and implemented to regulate their working hours, incentives and holidays.

\section{CARDIAC AUTONOMIC REGULATION IN RELATION TO OCCUPATIONAL PHYSICAL ACTIVITY AND PSYCHOSOCIAL JOB RESOURCES: A STUDY PROTOCOL}

J Vandeneede*, E Clays. Ghent university, Public Health, Ghent, Belgium

\subsection{6/oemed-2018-ICOHabstracts. 184}

Introduction In our modern service economies, considerable groups of workers remain exposed to high physical demands. Many jobs in Europe still involve manual labour and this proportion has hardly changed over the last years. In light of the growing evidence base on the harmful cardiovascular effects of occupational physical activity there is a need for developing preventive measures against premature cardiovascular morbidity and mortality in workers exposed to high physical demands. The aim of this study is to investigate objectively the relation between physically demanding jobs and the cardiovascular health of employees and the possible mediating role of psychosocial work factors.

Methods A field study including ambulatory registrations during 2-4 days of physical activity (PA), heart rate (HR) and heart rate variability (HRV) is conducted in a convenience sample of 450 employees. For optimising the external validity of the findings, the sample is not restricted to particular gender, job types or sectors of employment. Participants are recruited from workplaces within the manufacturing and service sector. Participation is on a voluntary basis with informed consent, and includes questionnaire assessment, medical examination (resting HR, blood pressure, and anthropometric measurements), a fitness test and a minimum of 48 hours field registration.

Result The HRV results will provide different insights in relation to workload, HR reserve (the ability to recover from work tasks) and the cardiovascular health. Given the study design, thorough assessment of confounders is required. Therefore, the baseline questionnaire includes standardised measures of socio-demographic information, physical and 